\title{
Prominent Lymphatic Vessel Hyperplasia with Progressive Dysfunction and Distinct Immune Cell Infiltration in Lymphedema
}

\author{
Epameinondas Gousopoulos, Steven T. Proulx, Jeannette Scholl, Maja Uecker, and Michael Detmar
}

From the Institute of Pharmaceutical Sciences, Swiss Federal Institute of Technology, ETH Zurich, Zurich, Switzerland

\author{
Accepted for publication \\ April 12, 2016. \\ Address correspondence to \\ Michael Detmar, M.D., Institute \\ of Pharmaceutical Sciences, \\ Swiss Federal Institute of \\ Technology, ETH Zurich, \\ Vladimir-Prelog-Weg 3, HCI \\ H303, CH-8093 Zurich, \\ Switzerland. E-mail: michael. \\ detmar@pharma.ethz.ch.
}

\begin{abstract}
Lymphedema is a common complication that occurs after breast cancer treatment in up to $30 \%$ of the patients undergoing surgical lymph node excision. It is associated with tissue swelling, fibrosis, increased risk of infection, and impaired wound healing. Despite the pronounced clinical manifestations of the disease, little is known about the morphological and functional characteristics of the lymphatic vasculature during the course of lymphedema progression. We used an experimental murine tail lymphedema model where sustained fluid stasis was generated on disruption of lymphatic flow, resulting in chronic edema formation with fibrosis and adipose tissue deposition. Morphological analysis of the lymphatic vessels revealed a dramatic expansion during the course of the disease, with active proliferation of lymphatic endothelial cells at the early stages of lymphedema. The lymphatic capillaries exhibited progressively impaired tracer filling and retrograde flow near the surgery site, whereas the collecting lymphatic vessels showed a gradually decreasing contraction amplitude with unchanged contraction frequency, leading to lymphatic contraction arrest at the later stages of the disease. Lymphedema onset was associated with pronounced infiltration by immune cells, predominantly $\mathrm{Ly}_{6 \mathrm{G}}{ }^{+}$and $\mathrm{CD}^{+}$cells, which have been linked to impaired lymphatic vessel function. (Am J Pathol 2016, 186: 2193-2203; http://dx.doi.org/10.1016/j.ajpath.2016.04.006)
\end{abstract}

The lymphatic vascular system has for a long time been a rather neglected research topic, which has delayed the appreciation of its role in cellular and physiological processes. ${ }^{1}$ Only recently, the discovery of lymphatic-specific genes has enabled a deeper understanding of mechanisms that control lymphatic development, and has helped to unravel an unanticipated role of lymphatic vessels in a large number of pathologies, including cancer progression, inflammation, and cardiovascular disease. ${ }^{2,3}$ Functional impairment of the lymphatic system results in reduced drainage of extravasated fluid and macromolecules, leading to a condition known as lymphedema, which most commonly occurs in the subcutaneous space. ${ }^{4}$

Lymphedema represents a complex pathobiology, where lymphatic insufficiency results in chronic fluid stasis with subsequent disruption of the normal cutaneous tissue architecture and profound inflammation. More important, lymphedema is characterized by increasing deposition of adipose tissue and progressive tissue fibrosis..$^{5}$ Secondary lymphedema represents the most common form of lymph drainage failure in Western countries, and occurs mainly as an iatrogenic complication after breast cancer surgery or surgical removal of other solid tumors, such as gynecological cancers and melanomas. ${ }^{6,7}$ The progressive swelling of the affected limb because of lymph stagnation and fibroadipose tissue deposition leads to a feeling of heaviness, functional impairment, sensory deficits, recurrent inflammation and infections, impaired wound healing, and, rarely, secondary tumors. 8,9

There is much evidence that lymphedema is a multistep procedure where lymphatic injury merely constitutes the initial trigger of the disease. ${ }^{10,11}$ This is supported by the

Supported by Swiss National Science Foundation grant 310030B_147087, European Research Council grant LYVICAM, Oncosuisse, Krebsliga Zurich (M.D.), and the ETH Zurich grant ETH-47 13-1 (M.D. and E.G.)

Disclosures: None declared. 
finding that only a fraction of the patients treated surgically for breast cancer develop lymphedema, which usually appears months to years after the surgery, indicating that secondary events are necessary to trigger the structural and functional deficits of this disorder, or that some patients are more prone to develop the condition. Some patients subjected to minimally invasive procedures (eg, lymph node biopsy) may develop lymphedema as well, suggesting that even minor lymphatic damage can initiate the pathobiologic sequence resulting in lymphedema.

Even though lymphatic injury is considered as the initiator of the pathological process, little attention has been paid to the structural and functional alterations of the lymphatic vasculature during lymphedema development. Lymphatic function is mainly evaluated by lymphoscintigraphy that provides mostly qualitative rather than quantitative results, ${ }^{12}$ whereas the quantitative evaluation of lymphatic contractile function has been limited. ${ }^{13}$

Lymphatic vessels dynamically participate in inflammatory reactions, modulate immune responses and immune tolerance, and respond to increased fluid loads in the tissue. They expand in inflammation, and their activation reduces the severity of tissue inflammation, ${ }^{14}$ highlighting an emerging role of the lymphatic vasculature as a therapeutic target. Inflammation also constitutes a hallmark of lymphedema in the clinic as well as in experimental models, ${ }^{5,15}$ and it has been reported that cyclooxygenase 2 inhibitors reduce the severity of experimental secondary lymphedema, indicating a potential involvement of inflammation in lymphedema development. ${ }^{16}$

Our aim was to investigate in detail the morphological and functional changes of lymphatic vessels in response to sustained fluid stasis in a surgically induced secondary lymphedema model. Surgical removal of a circumferential piece of mouse tail skin, together with ablation of deep collecting lymphatic vessels, was performed to reliably induce tissue swelling and lymphedema. We found that lymphedema development led to lymphatic vessel remodeling, with enlargement of these vessels and pronounced proliferation of lymphatic endothelial cells at the early stage of the disease. Functional in vivo studies using quantitative near-infrared imaging revealed a gradual impairment of collecting lymphatic vessel function and also a reduced perfusion of the lymphatic capillaries. Immunofluorescence and flow cytometry studies identified an increased immune cell infiltration with a predominant presence of Ly6G- and CD4-positive cells in the developing lymphedema.

\section{Materials and Methods}

\section{Lymphedema Mouse Tail Model}

Female C57BL/6J:ICR and $F V B$ mice were kept under conventional conditions. Surgery was performed at the age of 8 to 12 weeks. Tail lymphedema was induced by surgery, as previously described. ${ }^{17}$ In brief, a circumferential fullthickness portion of skin, 3 to $4 \mathrm{~mm}$, was excised $2 \mathrm{~cm}$ distal to the tail base. The deep collecting lymphatic vessels, running along the two lateral veins, were identified under a dissecting microscope by injecting 5 to $10 \mu \mathrm{L}$ of $1 \%$ Evans Blue Dye into the tip of the tail. They were microsurgically ablated, sparing the veins. Lymphedema development was studied for up to 6 weeks after the operation. All animal experiments were performed in accordance with animal protocols approved by the Cantonal Veterinary Office Zurich (license number 225/2013).

\section{Tail Volume Measurements and Histological Analyses}

Tail volume measurements were performed weekly, using a digital caliper, at $1-\mathrm{cm}$ intervals starting distally to the surgical excision site. Tail volumes were calculated using the truncated cone formula. ${ }^{18}$ For histological analysis, a 2- to 3-mm wide tail piece was dissected distally to the surgical excision margin and was fixed in $4 \%$ paraformaldehyde. The tissue was decalcified for 7 days using $0.5 \mathrm{~mol} / \mathrm{L}$ EDTA (Sigma, St. Louis, MO), and was then embedded in paraffin. Sections of $6 \mu \mathrm{m}$ were obtained, and histological analyses were performed on hematoxylin and eosin and picrosirius red stained sections. Sections were stained with hematoxylin solution (J.T. Baker, Center Valley, PA) for 3 minutes, washed under tap water, dipped in $0.1 \% \mathrm{HCl}$, and stained with eosin solution (J.T. Baker) for 1 minute. Other sections were stained with picrosirius red solution (Sigma) for 1 hour and then dipped in acidified water $(0.5 \%$ acetic acid in water). Sections were mounted with Eukitt medium (Sigma).

Immunofluorescence stains were performed on tail skin embedded in OCT (Sakura Finetec, Zoeterwoude, the Netherlands) and snap frozen in liquid nitrogen. Cryosections (7 $\mu \mathrm{m}$ thick) were fixed for 2 minutes in acetone $\left(-20^{\circ} \mathrm{C}\right)$ and 5 minutes in methanol $\left(4^{\circ} \mathrm{C}\right)$, washed in phosphatebuffered saline (PBS), incubated with immunomix (5\% nonimmune donkey serum and $0.2 \%$ bovine serum albumin in PBS with $0.3 \%$ Triton $\mathrm{X})$ and incubated overnight $\left(4^{\circ} \mathrm{C}\right)$ with rabbit anti-LYVE-1 antibody (AngioBio, San Diego, CA; 1:600), rat-Meca32 (BD Pharmingen, Franklin Lakes, $\mathrm{NJ}$; 1:200), or antibodies against Ki-67 (Dako, Glostrup, Denmark; 1:200), CD45 (R\&D Systems, Minneapolis, MN; 1:100), F4/80 (Abcam, Cambridge, UK; 1:200), CD68 (Abcam; 1:200), or CD206 (R\&D; 1:200). The sections were then incubated for 30 minutes with Alexa488- (1:200), Alexa594- (1:200) or Alexa350- (1:200) conjugated secondary antibodies and Hoechst 33342 (1:1000) (all from Invitrogen, Thermo Fischer, Waltham, MA). Some sections were fixed with $4 \%$ paraformaldehyde and then stained with BODIPY dye 493/503 (Invitrogen; 1:1000) in Dako diluent (Dako).

Immunofluorescence stains were analyzed using an Axioskop 2 mot plus microscope (Carl Zeiss, Oberkochen, Germany) equipped with an Illuminator HXP 120 (V) and an AxioCam MRc camera, and five images per tissue section were acquired with a Plan Apochromat $10 \times / 0.45$ 
numerical aperture objective. Images of hematoxylin and eosin and picrosirius red stained sections were acquired using a Slide Scanner Panoramic 250 BF Flash (3D Histech, Ramsey, NJ) equipped with a Brightfield CIS 3CCD VCCF52U25CL with a $20 \times / 0.8$ numerical aperture objective. Morphometric analyses of vessels and of epidermal thickness, as well as quantification of tissue immunofluorescence stains, were performed using ImageJ software version 1.46r (NIH, Bethesda, MD).

\section{Light Sheet Microscopy of Tail Tissues}

Mouse tail cross sections ( 4 to $5 \mathrm{~mm}$ thick) were fixed for 2 hours at $4{ }^{\circ} \mathrm{C}$. Samples were then permeabilized with $0.5 \%$ Triton X-100 in PBS for 2 days, washed, and blocked with PermBlock solution (1\% bovine serum albumin, $0.1 \%$ Tween 20) for 2 days (room temperature). Rabbit antiLYVE-1 (AngioBio) and rat anti-CD31 antibodies were used for staining, by incubation in PermBlock solution for 7 days at $4^{\circ} \mathrm{C}$ under constant agitation. After multiple washing steps in PBS with $0.1 \%$ Tween 20 for 2 days, the samples were incubated for 7 days in PermBlock with Alexa488- and Alexa594-conjugated antibodies at $4^{\circ} \mathrm{C}$ (Invitrogen). Tissue samples were then embedded in $1 \%$ ultrapure agarose gels on ice and dehydrated in a series of methanol solutions $(50 \%, 70 \%, 95 \%, 100 \%)$, each step for more than an hour with the last step overnight. Tissue clearing was achieved with incubation in $50 \%$ benzyl alcohol/benzyl benzoate (Acros Organics, Thermo Fischer; 1:2) for 3 to 4 days, until the tissue was visibly clear. Samples were stored in 50\% benzyl alcohol/benzyl benzoate at $4^{\circ} \mathrm{C}$ in the dark. Whole mount image acquisition was performed using an Olympus MVX10 LaVision BioTec Ultramicroscope (LaVision, Bielefeld, Germany), and images were processed using Imaris 7.6.4 (Bitplane).

\section{Flow Cytometry}

Mice were euthanized, and single-cell suspensions were obtained from the full-thickness tail skin distal to the surgical margin. The tissue was minced and incubated in $5 \mathrm{mg} / \mathrm{mL}$ collagenase II and $0.05 \mathrm{mg} / \mathrm{mL}$ DNase (both from Sigma) in RPMI 1640 medium (Gibco, Thermo Fischer) for 30 minutes at $37^{\circ} \mathrm{C}$. Single-cell suspensions were filtered through $70-$ and $40-\mu \mathrm{m}$ cell strainers and resuspended in FACS buffer ( $2 \%$ fetal bovine serum, $2 \mathrm{mmol} / \mathrm{L}$ EDTA). Cells were incubated with CD16/CD32 (1:100; Biolegend, San Diego, CA) to block endogenous Fc receptors and live/ dead staining was performed using Fixable Aqua Zombie (1:300; Invitrogen). Cell populations were analyzed using multicolor surface markers for myeloid and lymphoid populations. Myeloid panel: CD45 (Pacific blue, 1:400), Ly6G (fluorescein isothiocyanate, 1:250), Ly6C (PE, 1:125), CD11b (PerCPC5.5, 1:400), CD11c (PECy7, 1:400) (all from Biolegend), and F4/80 (Alexa Fluor 647; AbD Serotec, Oxford, UK; 1:400). Lymphocyte panel: CD45 (Pacific blue, 1:400), CD3 (PECy7, 1:200), CD4 (APC, 1:200), CD8 (fluorescein isothiocyanate, 1:400) (all from Biolegend).

\section{In Vivo Imaging of Lymphatic Vessel Function}

A Zeiss StereoLumar V12 stereomicroscope adapted for near infrared imaging (NIR) was used for lymphatic vessel imaging. ${ }^{19}$ Using a Harvard Apparatus PHD2000 syringe pump (Harvard Bioscience, Holliston, MA) and a custommade intradermal catheter consisting of PE10 tubing and a $30 \mathrm{~g}$ needle, two different protocols for bolus injections were applied. For visualization of the transport function in a region of interest near the surgical excision margin, a controlled infusion of $5 \mu \mathrm{L}$ of NIR dye P20D800 was performed at $0.5 \mu \mathrm{L} /$ minute for 10 minutes at a site $1 \mathrm{~cm}$ proximal from the tail tip. Videos were acquired for a total of 20 minutes. For quantification of collecting lymphatic vessel contractility, a bolus injection of $5 \mu \mathrm{L}$ of P20D800 dye was performed, followed by controlled infusion of an additional $1 \mu \mathrm{L}$ at a rate of $0.2 \mu \mathrm{L} /$ minute for 5 minutes, injected $1 \mathrm{~cm}$ proximal from the tail tip. During the controlled infusion, a 15-minute video recording took place at a location 2 to $3 \mathrm{~cm}$ proximal to the infusion site. At the end of the 15-minute video recording, an area ranging from the injection site to the surgical excision margin was imaged with serial snapshots. On the video recording, a region of interest analysis of fluorescence intensity was performed, using Zeiss AxioVision software Vs40 version 4.8.2.0 (Zeiss, Jena, Germany) to evaluate the contractility of the collecting lymphatic vessels. ${ }^{20} \mathrm{~A}$ custom software in Matlab was used to analyze the frequency and amplitude of the contractions for a period of 5 minutes (from 6 to 11 minutes of the 15-minute video). The contraction amplitude was expressed as a percentage of the mean signal intensity.

\section{Statistical Analysis}

All data represent means \pm SD. All experiments were conducted at least in three replicates. Means of two groups were compared with the two-tailed Student's $t$-test; means of three or more groups were analyzed with one-way analysis of variance with Tukey's post hoc multiple comparison test. Analyses were performed using GraphPad Prism version 6.0 (GraphPad Software, San Diego, CA). $P<0.05$ was accepted as statistically significant.

\section{Results}

\section{Surgical Induction of Lymphedema Results in Fibroadipose Tissue Accumulation}

We used an established mouse tail model of lymphedema, where lymphedema was surgically induced by removal of a circumferential portion of skin $2 \mathrm{~cm}$ distal to the tail base and dissection of the collecting lymphatic vessels. ${ }^{21}$ Mice were observed for 6 weeks postoperatively to evaluate the 
A
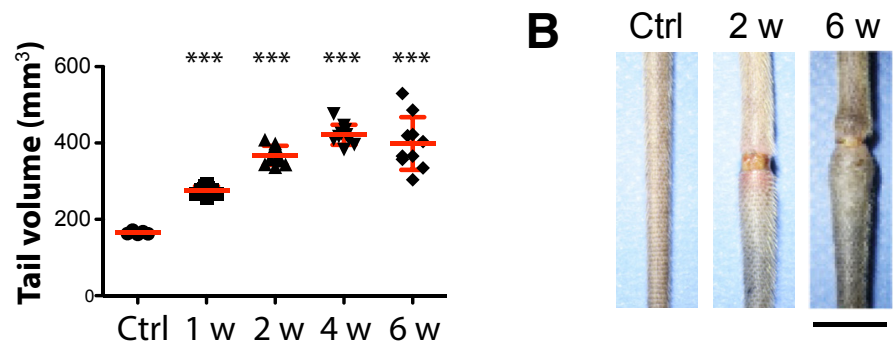

C Ctrl

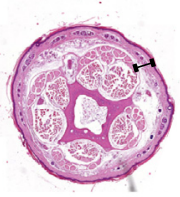

D

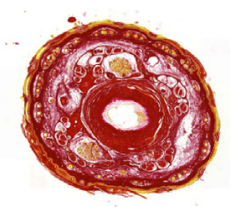

E

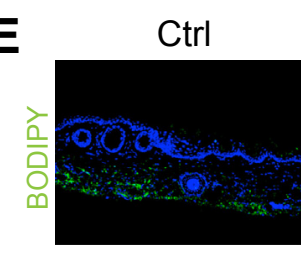

$\mathbf{F}$

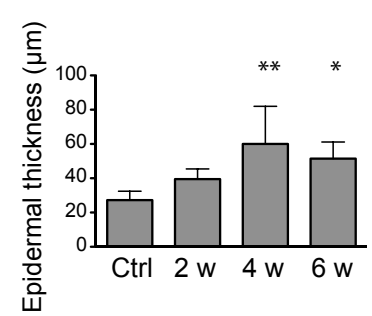

$2 w$
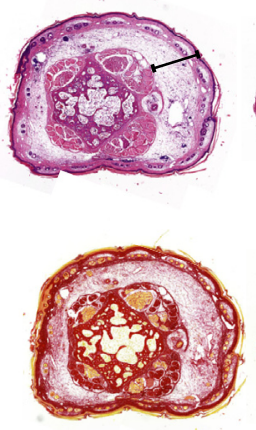

$2 w$

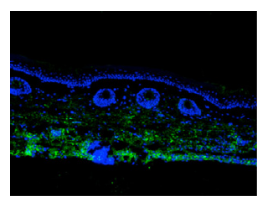

G
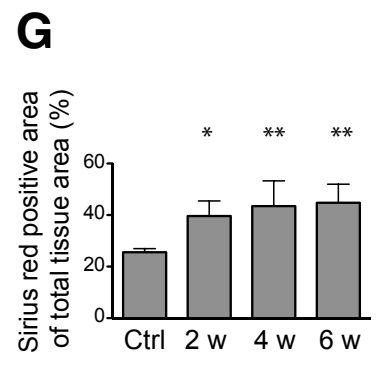

$4 \mathrm{w}$
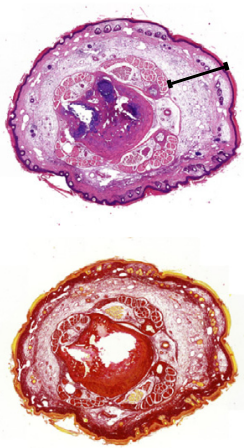

$4 w$

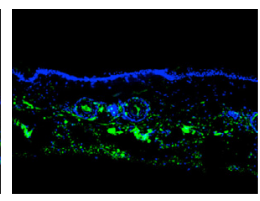

H
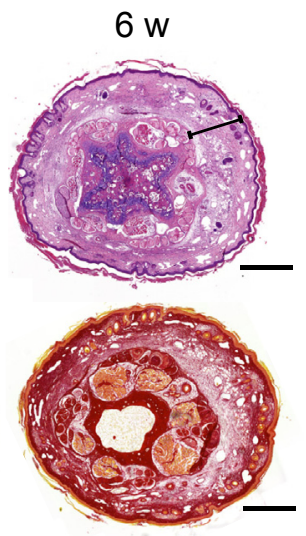

$6 \mathrm{w}$
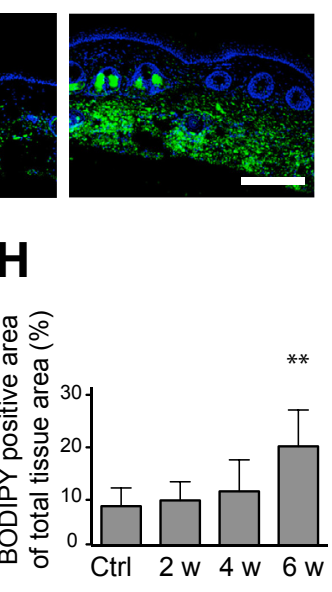

Figure 1 Fibroadipose tissue accumulation in lymphedema. A: Increase of tail volume over the course of lymphedema development. B: Representative images of unoperated control (Ctrl), 2 weeks $(w)$ and 6 weeks postoperative mouse tails. C: Hematoxylin and eosin (H\&E) stains of paraffin tail cross sections obtained 0.5 to $1 \mathrm{~cm}$ distal to the surgical excision margin. Increased tissue edema, enlarged lymphatic vessels, and inflammation in the operated samples. The drawn line indicates the extent of edema formation. D: Sirius red staining of paraffin tail cross sections reveals collagen in red. Collagen deposition was evaluated between the epidermis and the muscle fascia. $\mathbf{E}$ : Cryosections (7 $\mu \mathrm{m}$ thick) of mouse tails were stained for lipid droplets with BODIPY. F: Epidermal thickness was quantified from H\&Estained tail cross sections. G: Collagen quantification was performed by evaluating the collagen deposition between the epidermis and the muscle fascia (edematic area) in Sirius red-stained sections. H: BODIPY staining was quantified as percentage of the total tissue (section) area. ${ }^{*} P<0.05,{ }^{* *} P<0.01$, and ${ }^{* * *} P<0.001$ versus the control (one-way analysis of variance with Tukey's multiple comparison test). Scale bars: $1 \mathrm{~cm}$ (B); $1 \mathrm{~mm}$ (C and D); $200 \mu \mathrm{m}$ (E). course of lymphedema development. Evaluation of the tail volume distal to the surgical incision area showed a gradual increase that started 1 week postoperatively (1.6-fold change versus control unoperated mice, $P<0.001)$ and steadily increased until week 6 (2.4-fold change versus control, $P<0.001$ ) (Figure 1, A and B). Whole-tail cross sections stained with hematoxylin and eosin revealed several architectural changes of the edematic tails, with increased edema, cellularity, and epidermal thickness in comparison to unoperated tails (Figure 1C). The epidermal thickness increased from $27.3 \pm 5.2 \mu \mathrm{m}$ to $60.1 \pm 21.9 \mu \mathrm{m}$ after 4 weeks $(P<0.01)$ and to $51.4 \pm 9.7 \mu \mathrm{m}$ after 6 weeks $(P<0.05)$ (Figure $1 \mathrm{~F})$. Fibrotic tissue deposition was assessed by quantification of collagen using sirius red staining, which revealed an increased collagen deposition (1.5-fold increase compared with control tails, $P<0.05)$ that began 2 weeks postoperatively with a further increase after 4 and 6 weeks (Figure 1, D and G). Lipid accumulation, evaluated with BODIPY staining, revealed lipid deposition that was significantly increased at 6 weeks postoperatively (2.6-fold increase compared with control tails, $P<0.01$ ) (Figure 1, E and $\mathrm{H}$ ). These results indicate that the used mouse tail model faithfully recapitulated several of the clinical features of lymphedema.

\section{Lymphatic Vessel Expansion during Lymphedema Development}

We next performed an evaluation of blood and lymphatic vessels using immunofluorescence stains for Meca-32 (blood vessel marker) and LYVE-1 (lymphatic vessel marker). No major changes of blood vascularity were found 
A

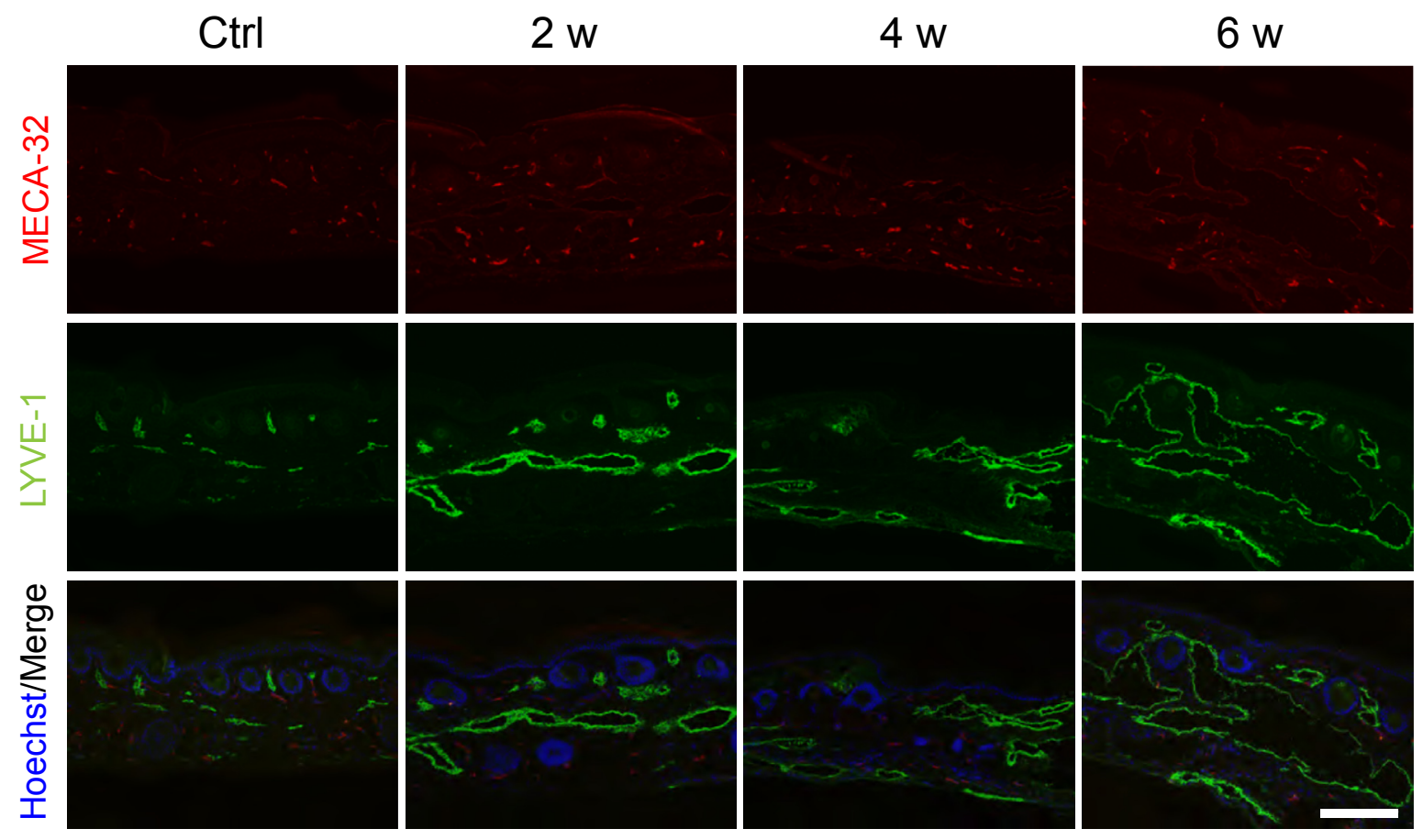

B

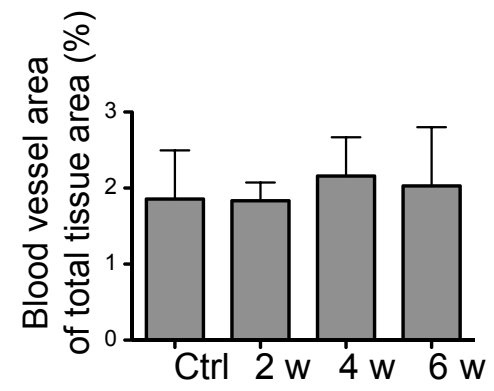

C

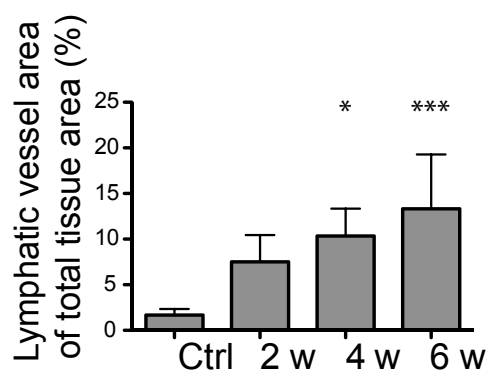

D

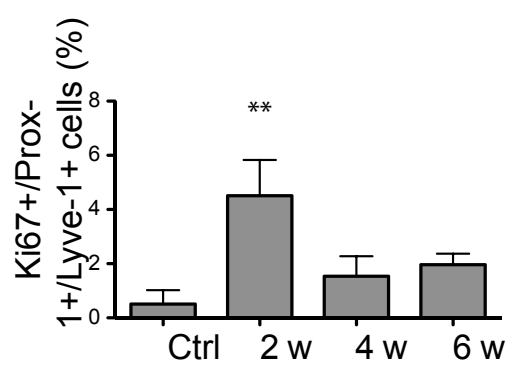

\section{E}
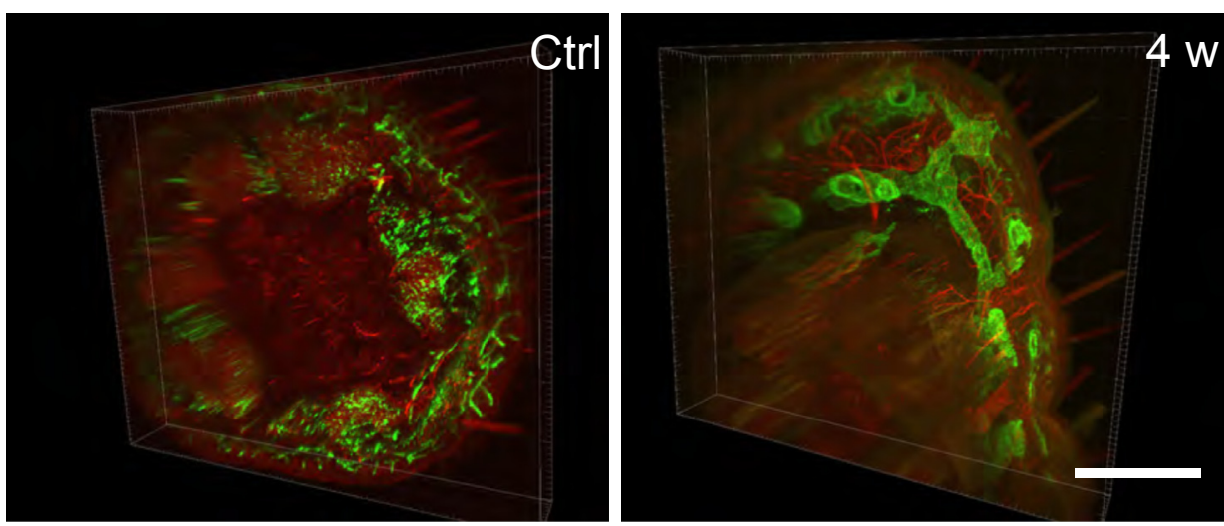

Figure 2 Lymphatic dilation and lymphangiogenesis in lymphedema. A: Immunofluorescence stains for lymphatic (Lyve-1) and blood (Meca-32) vessels of control (Ctrl) and operated mice. Quantification of blood vessel area (B) and lymphatic vessel area (C) during the course of lymphedema. D: Quantification of Prox$1^{+} /$Lyve $-1^{+} / \mathrm{Ki}-67^{+}$cells reveals proliferation of lymphatic endothelial cells at the early stage [2 weeks (w) postoperatively] of lymphedema. E: Representative three-dimensional light sheet microscopic image illustrates the morphological changes of lymphatic vessel in lymphedema. ${ }^{*} P<0.05,{ }^{* *} P<0.01$, and ${ }^{* * *} P<0.001$ versus control (one-way analysis of variance with Tukey's multiple comparison test). Scale bars: $200 \mu \mathrm{m}(\mathrm{A}) ; 1 \mathrm{~cm}(\mathrm{E})$. 

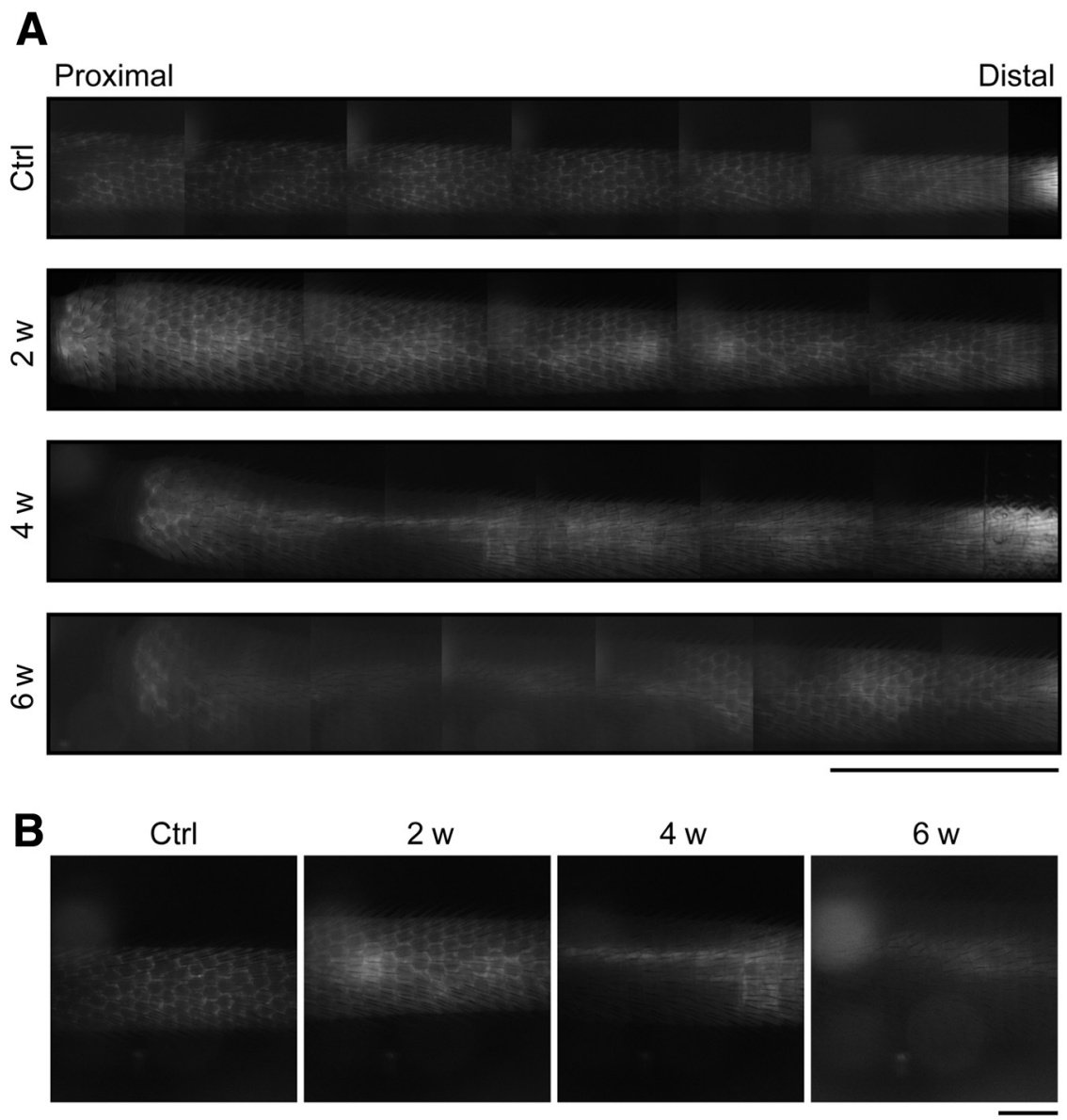

Figure 3 Gradual impairment of lymphatic capillary function during lymphedema development. A: Whole tail reconstruction using snapshot stereomicroscope images 15 minutes after injection of the near infrared imaging dye. Gradual disappearance of the honeycomb lymphatic capillary pattern during lymphedema development, indicating decreased perfusion of the lymphatic capillary vessels. B: Loss of the honeycomb pattern of lymphatic capillaries and interstitial accumulation of the injected dye in tail close-ups located 1 to $2 \mathrm{~cm}$ distal to the surgical excision margin. Scale bars: $1 \mathrm{~cm}$ (A); $2 \mathrm{~mm}$ (B). Ctrl, control; w, weeks. during the 6 postoperative weeks (Figure 2, A and B). In contrast, there was a profound enlargement of lymphatic capillaries in comparison to the control unoperated mice (Figure 2A). We found that the lymphatic vessel area gradually increased during the course of edema development and at 6 weeks postsurgery accounted for $13.3 \% \pm 5.9 \%$ of total tissue area in comparison to $1.6 \% \pm 0.7 \%$ in the control tissue $(P<0.001)$ (Figure $2 \mathrm{C}$ ).

We next investigated whether the lymphatic vessel expansion was the result of passive dilation because of fluid accumulation or if the enlargement was caused by active proliferation of lymphatic endothelial cells. Quantitative analysis of lymphatic endothelial cell proliferation using triple immunofluorescence staining for the lymphatic markers Lyve- 1 and Prox-1 and for the proliferation marker Ki-67 identified a significant increase $(4.5 \% \pm 2.9 \%$ versus $0.5 \% \pm 1.1 \% ; P<0.01)$ in the percentage of $\mathrm{Ki}-67^{+}$ lymphatic endothelial cells (Lyve- $1^{+}$Prox $-1^{+}$) at the early stage (2 weeks postoperatively) of lymphedema (Figure 2D). There was a nonsignificant trend for increased proliferation of lymphatic endothelial cells at 4 and 6 weeks after surgery. Visualization of the three-dimensional lymphatic vessel architecture using light-sheet microscopy revealed profound structural changes of the lymphatic vasculature on induction of lymph stasis, with an enlarged and plump appearance of the lymphatic capillaries (Figure 2E). These results indicate that active lymphatic vessel expansion occurs during the course of lymphedema development.

\section{Gradual Functional Impairment of Lymphatic Collectors and Capillaries during Lymphedema Development}

To evaluate the functionality of the lymphatic vasculature during the course of lymphedema development, we used near-infrared intravital microscopy, as previously described. ${ }^{19}$ Using an infusion pump and an intradermal catheter, the lymphatic-specific tracer P20D800 was injected close to the distal end of the tail and the uptake and transport of the tracer through the initial and collecting lymphatic vessel network was monitored by stereomicroscopic nearinfrared imaging. In unoperated control mice, there was a rapid transport of the tracer toward the tail base via lymphatic collectors and the capillary network, which appeared with a regular pattern of honeycomb-like structures (Figure 3A and Supplemental Video S1). At 2 weeks postoperatively, the transport of the tracer toward the tail base was delayed and retrograde flow through lymphatic capillaries occurred close to the excision site (Supplemental Video S2). Nonetheless, we still observed a complete filling of the lymphatic capillaries with tracer 15 minutes after 

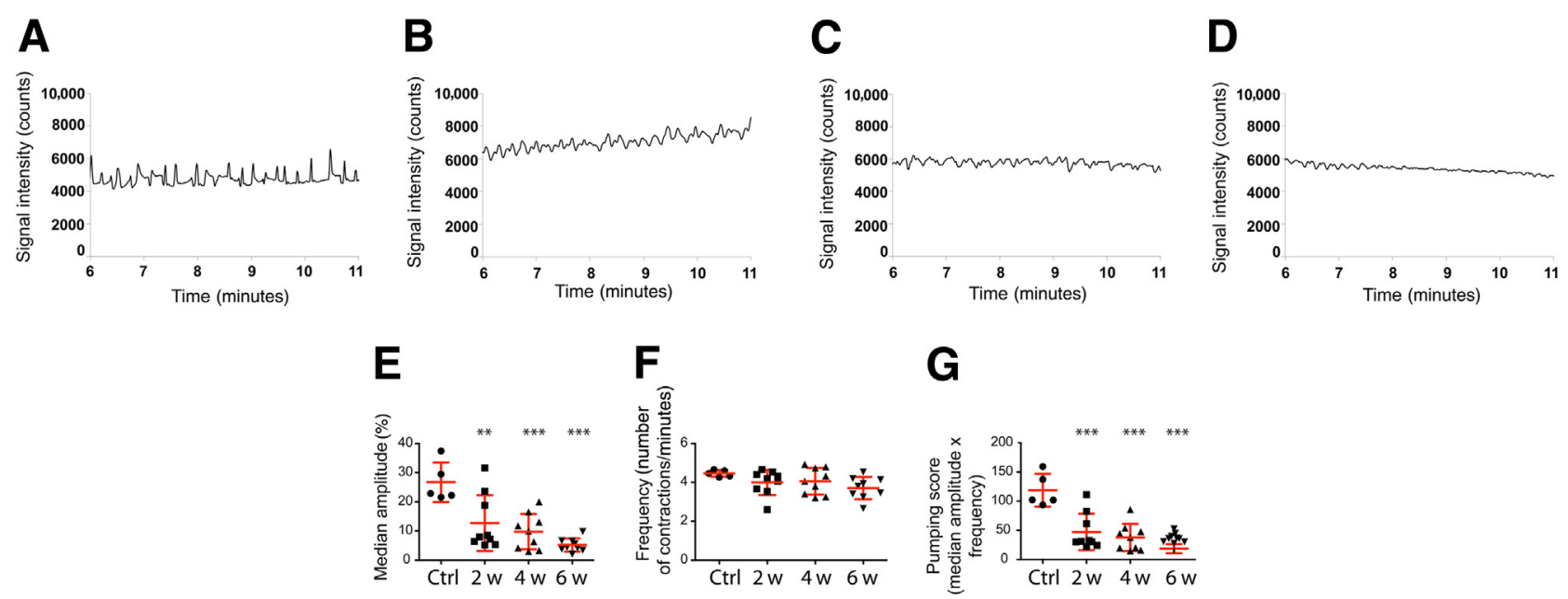

Figure 4 Quantitative assessment of collecting lymphatic vessel contractility of unoperated mice and of lymphedema mice at 2, 4, and 6 weeks (w) after the surgery. A: Representative contractility pattern of nonoperated tail, showing large-amplitude contractions. B: Representative contractility pattern of tail 2 weeks after surgery, showing preservation of contractility albeit with smaller-amplitude contractions. C: Representative contractility pattern of tail 4 weeks after surgery, showing a further decrease in contraction amplitude. D: Representative contractility pattern of tail 6 weeks after surgery, showing only weak amplitude contractions. E: Quantification of contraction amplitudes reveals a significant decrease at all postoperative time points. F: Quantification of the frequency of contractions reveals no significant changes after induction of lymphedema. G: Quantification of pumping score (amplitude $\times$ frequency) reveals a significant decrease at each postoperative time point. ${ }^{* *} P<0.01,{ }^{* *} P<0.001$ (one-way analysis of variance with Tukey's multiple comparison test). Ctrl, control.

injection (Figure 3A). At 4 and 6 weeks after surgery, the transport time to the incision area was further delayed and a discontinuous uptake of the tracer into the capillary network was observed (Figure 3A and Supplemental Video S3). There was also an increased amount of tracer detected in the interstitial tissue. Close-ups of control and operated tails demonstrated the gradual loss of the perfused honeycomb pattern of lymphatic capillaries over time in tails with lymphedema (Figure 3B).

We next assessed the functionality of the collecting lymphatic vessels by analyzing the contractility of these vessels during near-infrared video acquisitions. By using a region of interest analysis on each collecting vessel, we were able to visualize and quantify the contraction amplitude and frequency. Unoperated mice demonstrated a regular pattern of contractions with a large amplitude (Figure 4A and Supplemental Video S4). Starting at 2 weeks postoperatively, the amplitude was diminished but the frequency was unaffected (Figure 4B and Supplemental Video S5). After 4 weeks, the contraction amplitude was even more blunted (Figure 4C and Supplemental Video S6), and at 6 weeks after the operation, it was barely detectable (Figure 4D and Supplemental Video S7). A quantitative analysis revealed a significantly reduced amplitude of the lymphatic vessel contractions at each time point after lymphedema induction (control: $26.7 \% \pm 6.8 \%$; 2 weeks: $12.7 \% \pm 9.5 \%$; 4 weeks: $9.8 \% \pm 6.1 \%$; 6 weeks: $5.1 \% \pm 2.3 \%$, expressed as a percentage of mean signal intensity), whereas the frequency of the contractions did not significantly change during the disease progression in comparison to unoperated controls (control: $4.5 \pm 0.1 ; 2$ weeks: $4.0 \pm 0.6$; 4 weeks: $4.1 \pm 0.7 ; 6$ weeks: $3.7 \pm 0.6$ contractions/minute) (Figure 4, E and F). The pumping score, which was calculated by multiplying the contraction amplitude with the contraction frequency, was $118.9 \pm 28$ in unoperated mice (Figure $4 \mathrm{G}$ ) and declined significantly on induction of lymphedema ( 2 weeks: $47.3 \pm 31.2 ; 4$ weeks: $37.8 \pm 23.2 ; 6$ weeks: $18.7 \pm 7.7$ ). These results indicate a progressive loss of function of both initial and collecting lymphatic vessels during the development of lymphedema in this model.

\section{Distinct Changes in the Immune Cell Infiltrate in Lymphedema}

Lymphedema has been described to be associated with an increased immune cell infiltrate. ${ }^{22}$ Immunofluorescence stains revealed a slight increase in the infiltration by $\mathrm{CD} 45^{+}$ cells at 2 weeks postoperatively, with a significant increase at 4 weeks and even more pronounced at 6 weeks after the operations (control: $1.01 \% \pm 0.42 \%$ of the tissue area; 2 weeks: $4.18 \% \pm 1.37 \%$; 4 weeks: $6.70 \% \pm 3.58 \%$; 6 weeks: $9.11 \% \pm 1.84 \%$ ). Infiltration by $\mathrm{F} 4 / 80^{-}$and CD68-positive macrophages, as well as by CD206-positive cells, reached a peak at 4 weeks postoperatively (Figure 5). To more precisely quantify these different immune populations at the onset of the disease, flow cytometry analysis was performed on mice 2 weeks after the operation (Figure 6). There was a significant increase of $\mathrm{CD}^{4} 5^{+}$cells from $0.41 \% \pm 0.07 \%$ of the total single cells in unoperated mouse tails to $2.05 \% \pm 1.03 \%$ at 2 weeks postoperatively $(P<0.01)$ (Figure 6A). Further analysis of other immune cells subpopulation revealed an increase of both myeloid and lymphoid cells 2 weeks postoperatively, with $\mathrm{Ly}_{6 \mathrm{G}}{ }^{+}$and $\mathrm{CD}^{+}$cells accounting for most of the infiltrate $\left(\mathrm{Ly} 6 \mathrm{G}^{+}\right.$: from $0.006 \% \pm 0.001 \%$ to $0.9 \% \pm 0.6 \%, P<0.05 ; \mathrm{CD}^{+}$: 
A
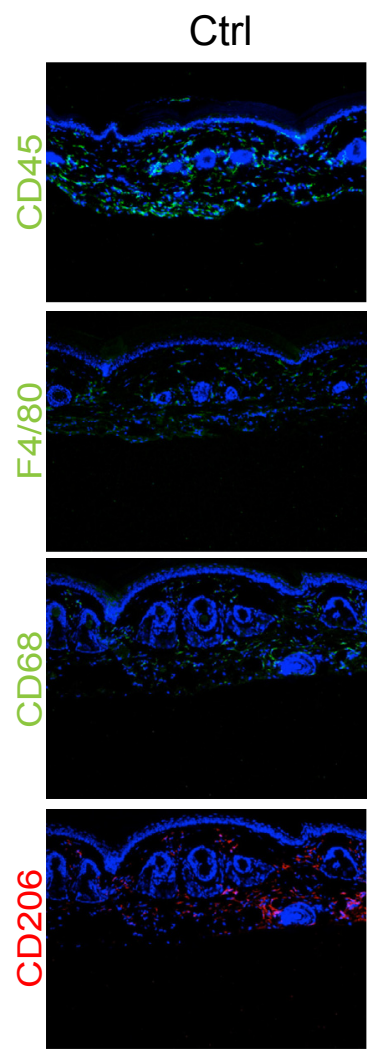

$2 \mathrm{w}$
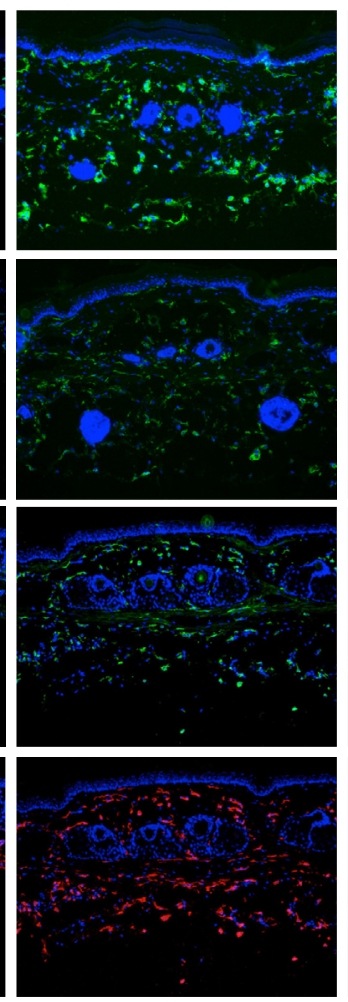

$4 \mathrm{w}$
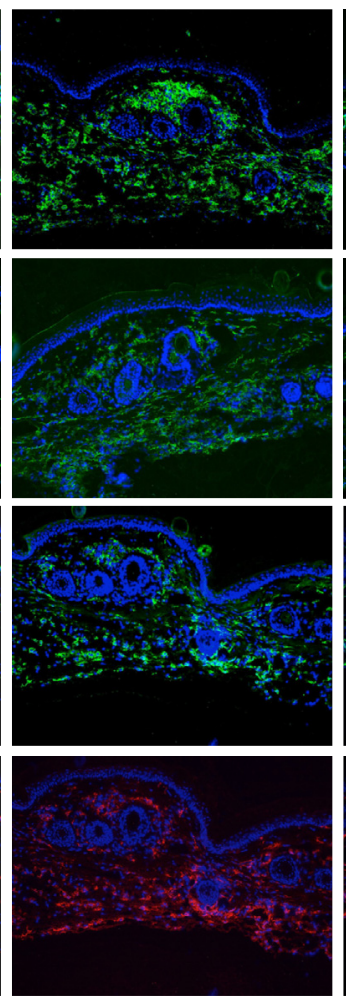
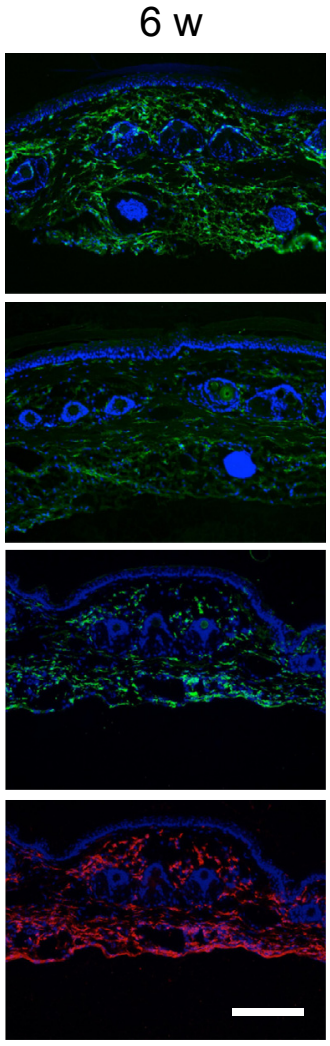

\section{B}

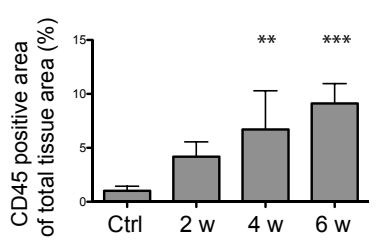

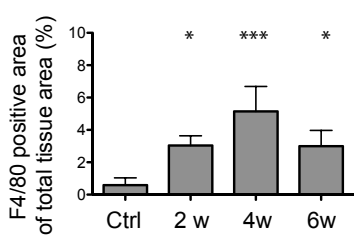
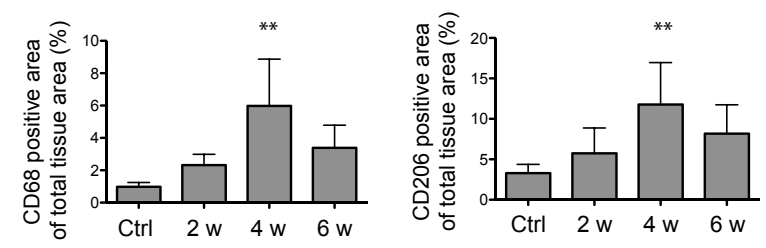

Figure 5 Increased immune cell infiltration during lymphedema progression. A: Immunofluorescence stains for immune cells markers (CD45, F4/80, CD68, CD206) demonstrate an increased infiltration of immune cells at 2, 4, and 6 weeks (w) postoperatively. B: Immunostains were quantified as percentage stained area of the total tissue area. ${ }^{*} P<0.05,{ }^{* *} P<0.01$, and ${ }^{* * *} P<0.001$ versus the control (one-way analysis of variance with Tukey's multiple comparison test). Scale bar $=200 \mu \mathrm{m}$. Ctrl, control.

from $0.02 \% \pm 0.008 \%$ to $0.5 \% \pm 0.2 \%$ of the total single cells; $P<0.05$ ) (Figure 6, B and D). Monocytes $\left(\right.$ Ly6 $\mathrm{C}^{+}$ cells), macrophages $\left(\mathrm{CD} 11 \mathrm{c}^{+} \mathrm{F} 4 / 80^{+}\right.$cells), and $\mathrm{CD} 8^{+}$ cells increased as well $\left(\mathrm{Ly}_{6 \mathrm{C}^{+}}\right.$: from $0.01 \% \pm 0.005 \%$ to $0.07 \% \pm 0.03 \%, P<0.05 ; \mathrm{CD} 11 \mathrm{c}^{+} \mathrm{F} 4 / 80^{+}$: from $0.04 \% \pm 0.01 \%$ to $0.39 \% \pm 0.08 \%, P<0.001 ; \mathrm{CD}^{+}$: from $0.002 \% \pm 0.001 \%$ to $0.04 \% \pm 0.01 \%, P<0.05$ ) (Figure 6, B-D). Thus, a pronounced increase in immune cell infiltration was apparent in lymphedema tails at 2 weeks after operation.

\section{Discussion}

In this study, we used a mouse tail model of lymphedema and performed immunofluorescence analyses and functional assays to analyze the morphology and functionality of lymphatic capillaries and collecting lymphatic vessels during the course of the disease, as well as the immune cell infiltration, with a particular focus at the early stage of lymphedema development. We found that lymphatic vessel morphology was profoundly altered during the course of lymphedema development and was associated with a gradual impairment of lymphatic vessel function and a distinct immune cell infiltration.

Secondary lymphedema is a rather frequent complication of cancer surgery and presents as a disease with complex pathology. It primarily develops because of the insufficiency of the lymphatic vasculature to drain the extravasated interstitial fluid and macromolecules. Clinically and histologically, lymphedema is characterized by edema formation manifested as swelling of the limb, epidermal thickening, adipose tissue accumulation, and fibrosis, which was initially described in primary lymphedema ${ }^{23}$ and has been 

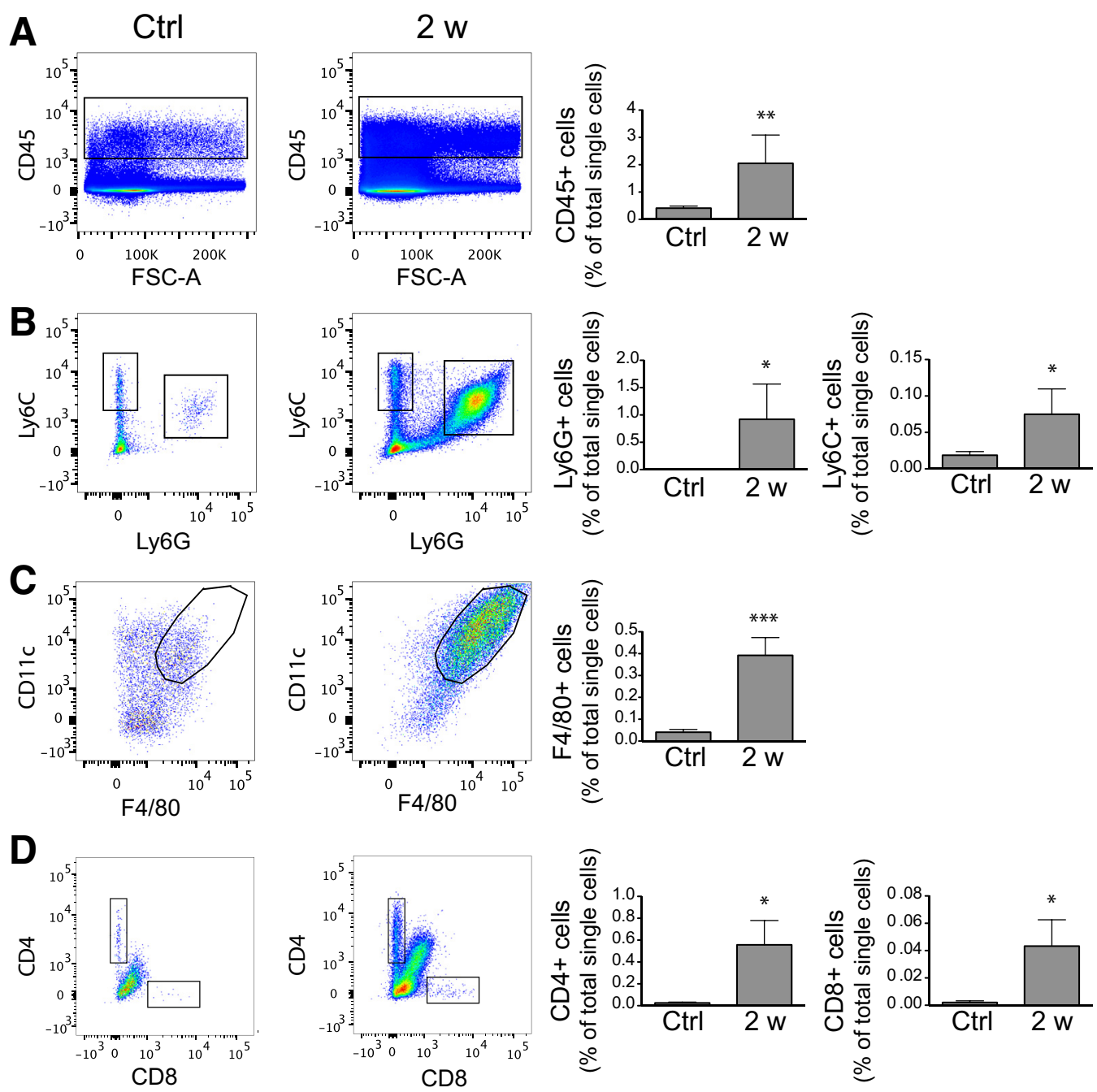

Figure 6 Characterization of immune cell infiltrate in lymphedema. Representative flow cytometry plots and quantification of CD45 ${ }^{+}$cells $(\mathbf{A})$, the main myeloid cells (B and C), and lymphocytes (D). There is an overall increase of inflammatory cells infiltrating the lymphedematic tissue 2 weeks (w) postoperatively, with predominant presence of $\mathrm{Ly}_{6 \mathrm{G}}{ }^{+}$and $\mathrm{CD}^{+}$cells. Quantification of cell populations is displayed as percentage of total single cells. Circled and boxed areas show the gating of the populations quantified in the accompanying graphs. ${ }^{*} P<0.05, * * P<0.01$, and ${ }^{* *} P<0.001$ (unpaired two-tailed $t$-test). Ctrl, control.

detected in secondary lymphedema as well. ${ }^{5}$ In our study, we found that tail swelling and fibroadipose tissue deposition were strongly induced on removal of a circumferential portion of skin and ablation of the collecting lymphatic vessels, thus reliably reproducing the histopathological hallmarks of the disease.

The lymphatic vasculature is an integral, although rather understudied, component of lymphedema development, often concealed by the pathological manifestations of the disease. Both dermal lymphatic hyperplasia and hypoplasia have been reported in lymphedema patients, ${ }^{24,25}$ where it has surprisingly been noted that dilation of the lymphatic capillaries occurs even in the contralateral arm of patients experiencing lymphedema. ${ }^{26}$ In the experimental mouse tail model, we observed a gradual enlargement of lymphatic vessels with an increase of the lymphatic vessel area during the course of lymphedema development, whereas the blood vascularity remained unchanged. Evaluation of lymphatic endothelial cell proliferation revealed an active proliferation of these cells, which was most prominent during the early stage of lymphedema development ( 2 weeks postoperatively). This proliferation of the lymphatic endothelial cells does not appear to lead to sprouting lymphangiogenesis but, instead, to the enlargement of existing lymphatic vessels, resulting in a profound remodeling of the lymphatic vasculature as demonstrated with the three-dimensional light sheet microscopy. Similar findings have been reported in chronic inflammation (eg, of the gastrointestinal tract), indicating that lymphatic vessel expansion may represent a compensatory mechanism to maintain interstitial fluid clearance and fluid homeostasis. ${ }^{27}$

When imaged through the skin surface, lymphatic capillaries in the mouse tail normally have a honeycomb-like pattern. ${ }^{17,28}$ Evaluation of the lymphatic capillaries in unoperated control mice with NIR imaging confirmed the honeycomb pattern, with rapid transport of an injected tracer through the initial and collecting lymphatic vessels toward 
the tail base. Surgical induction of lymphedema, however, resulted in a gradual impairment of lymphatic capillary function, identified as the loss of the intact honeycomb-like pattern and increased evidence of retrograde flow, particularly in close proximity to the surgical excision site. Although it remains a technical challenge to accurately measure pressure within lymphatic vessels in mice, increases in intralymphatic pressure, which have previously been reported within lymphatic capillaries in human primary lymphedema, are likely also present in our model and thus could inhibit the efficient filling of these vessels with the injected tracer. ${ }^{29}$ These findings suggest that interstitial fluid accumulation causes edema, leading to lymphatic vessel remodeling, which compromises the function of the lymphatic capillaries.

We also examined the functionality of the collecting lymphatic vessels by evaluating their contractility using noninvasive NIR imaging. Previous studies have reported a reduced frequency of contractions in experimental models of inflammation and cancer. ${ }^{19,30}$ Despite the absence of comprehensive clinical studies, two clinical reports of lymphedema patients have shown reduced contractility of lymphatic vessels and a negative correlation between the pressure generated by the lymphatic pump and the degree of swelling. ${ }^{13,25}$ In accordance with these clinical observations, our study demonstrates, for the first time, a gradual decrease of the collecting lymphatic vessel contractility during progression of lymphedema in an animal model. Significant decreases in contractile amplitude were seen already at 2 weeks postoperatively, and led to an almost complete lymphatic vessel arrest at the later stages of lymphedema. More important, unlike in other inflammatory conditions, ${ }^{19,31}$ reduced lymphatic vessel contractile amplitude/ strength was observed in lymphedema rather than a reduction in the frequency of contractions.

The peripheral lymphatic system regulates tissue fluid homeostasis and also directs the transport of immune cells. It has been unclear for a long time how the lymphatic vasculature is affected by inflammation. However, several recent studies have revealed that inflammation leads to lymphatic vessel expansion, with lymphatic hyperplasia and lymphangiogenesis in several inflammatory conditions, both in patients and in experimental models. ${ }^{32}$ It has also been found that inflammation is associated with impaired lymphatic drainage, particularly in chronic inflammatory conditions. ${ }^{30,33,34}$ Lymphedema shows pronounced inflammatory manifestations, ${ }^{22}$ but whether tissue inflammation is a consequence of or an active contributor to lymphatic vascular insufficiency has not been studied in much detail. It has been suggested that infiltration of nitric oxideproducing $\mathrm{CD}_{11 \mathrm{~b}^{+}} \mathrm{Gr}^{+}$cells on oxazolone skin sensitization might modulate the microenvironment surrounding the collecting lymphatic vessels, thus impairing lymphatic contractility. ${ }^{35}$ Similarly, CD11 $b^{+}$cells have been reported to contribute to reduced lymphatic contractility. ${ }^{31,33}$ In our study, we identified significantly increased populations of $\mathrm{CD} 11 \mathrm{~b}^{+} \mathrm{Ly}_{6 \mathrm{G}}{ }^{+}$cells, a subgroup of $\mathrm{CD} 11 \mathrm{~b}^{+} \mathrm{Gr} 1^{+}$cells, and $\mathrm{CD}_{4}^{+}$immune cells in the affected tissue 2 weeks postoperatively, a time point that coincides with the onset of the lymphatic vessel contractility decrease. It has been reported that depletion of $\mathrm{CD}^{+}$cells leads to improved lymphatic function in a murine lymphedema model. ${ }^{36}$ However, in another study in which all $\mathrm{CD}_{11} \mathrm{~b}^{+}$cells were depleted in a lymphedema model, ${ }^{37}$ no change in swelling or inflammation occurred. Thus, it is conceivable that a global $\mathrm{CD} 11 \mathrm{~b}^{+}$cell depletion results in elimination of both harmful and beneficial players in lymphedema progression. For example, macrophages may support lymphangiogenesis, ${ }^{38,39}$ and their depletion might counterbalance the depletion of adverse immune cell subsets.

Together, our data reveal, for the first time, an active role of the lymphatic vasculature in lymphedema development, indicating that monitoring of lymphatic transport function might serve as a clinical parameter to evaluate the disease onset/progression in postcancer surgery patients. The increased presence of specific immune cell populations at the early stages of lymphedema may offer insights into the pathobiology of the disease, which will have to be further studied, because they may constitute potential therapeutic targets to ameliorate lymphedema.

\section{Acknowledgments}

We thank Dr. Lothar Dieterich for valuable discussions and Catharina Seidel for advice regarding the light sheet microscopy technique.

E.G. and M.D. designed the study; E.G., S.T.P., J.S., and M.U. performed experiments and collected and analyzed data; E.G., S.T.P., J.S., M.U., and M.D. wrote the manuscript.

\section{Supplemental Data}

Supplemental material for this article can be found at http://dx.doi.org/10.1016/j.ajpath.2016.04.006.

\section{References}

1. McMaster PD: Lymphatic participation in cutaneous phenomena: Harvey Lecture, April 16, 1942. Bull N Y Acad Med 1942, 18: $731-767$

2. Alitalo K: The lymphatic vasculature in disease. Nat Med 2011, 17 : $1371-1380$

3. Huang LH, Elvington A, Randolph GJ: The role of the lymphatic system in cholesterol transport. Front Pharmacol 2015, 6:182

4. Mortimer PS, Rockson SG: New developments in clinical aspects of lymphatic disease. J Clin Invest 2014, 124:915-921

5. Rockson SG: Lymphedema. Am J Med 2001, 110:288-295

6. Cormier JN, Askew RL, Mungovan KS, Xing Y, Ross MI, Armer JM: Lymphedema beyond breast cancer: a systematic review and metaanalysis of cancer-related secondary lymphedema. Cancer 2010, 116: 5138-5149

7. Rockson SG, Rivera KK: Estimating the population burden of lymphedema. Ann N Y Acad Sci 2008, 1131:147-154 
8. Stewart FW, Treves N: Lymphangiosarcoma in postmastectomy lymphedema: a report of six cases in elephantiasis chirurgica. Cancer 1948, 1:64-81

9. Velanovich V, Szymanski W: Quality of life of breast cancer patients with lymphedema. Am J Surg 1999, 177:184-187; discussion 188

10. Modi S, Stanton AW, Mellor RH, Peters AM, Levick JR, Mortimer PS: Regional distribution of epifascial swelling and epifascial lymph drainage rate constants in breast cancer-related lymphedema. Lymphat Res Biol 2005, 3:3-15

11. Stanton AW, Svensson WE, Mellor RH, Peters AM, Levick JR, Mortimer PS: Differences in lymph drainage between swollen and nonswollen regions in arms with breast-cancer-related lymphoedema. Clin Sci (Lond) 2001, 101:131-140

12. Modi S, Stanton AW, Mortimer PS, Levick JR: Clinical assessment of human lymph flow using removal rate constants of interstitial macromolecules: a critical review of lymphoscintigraphy. Lymphat Res Biol 2007, 5:183-202

13. Modi S, Stanton AW, Svensson WE, Peters AM, Mortimer PS, Levick JR: Human lymphatic pumping measured in healthy and lymphoedematous arms by lymphatic congestion lymphoscintigraphy. J Physiol 2007, 583:271-285

14. Huggenberger R, Ullmann S, Proulx ST, Pytowski B, Alitalo K, Detmar M: Stimulation of lymphangiogenesis via VEGFR-3 inhibits chronic skin inflammation. J Exp Med 2010, 207:2255-2269

15. Frueh FS, Gousopoulos E, Rezaeian F, Menger MD, Lindenblatt N, Giovanoli P: Animal models in surgical lymphedema research: a systematic review. J Surg Res 2016, 200:208-220

16. Kashiwagi S, Hosono K, Suzuki T, Takeda A, Uchinuma E, Majima M: Role of COX-2 in lymphangiogenesis and restoration of lymphatic flow in secondary lymphedema. Lab Invest 2011, 91: $1314-1325$

17. Rutkowski JM, Moya M, Johannes J, Goldman J, Swartz MA: Secondary lymphedema in the mouse tail: lymphatic hyperplasia, VEGF-C upregulation, and the protective role of MMP-9. Microvasc Res 2006, $72: 161-171$

18. Sitzia J: Volume measurement in lymphoedema treatment: examination of formulae. Eur J Cancer Care (Engl) 1995, 4:11-16

19. Proulx ST, Luciani P, Christiansen A, Karaman S, Blum KS, Rinderknecht M, Leroux JC, Detmar M: Use of a PEG-conjugated bright near-infrared dye for functional imaging of rerouting of tumor lymphatic drainage after sentinel lymph node metastasis. Biomaterials 2013, 34:5128-5137

20. Blum KS, Karaman S, Proulx ST, Ochsenbein AM, Luciani P, Leroux JC, Wolfrum C, Detmar M: Chronic high-fat diet impairs collecting lymphatic vessel function in mice. PLoS One 2014, 9: e94713

21. Clavin NW, Avraham T, Fernandez J, Daluvoy SV, Soares MA, Chaudhry A, Mehrara BJ: TGF-betal is a negative regulator of lymphatic regeneration during wound repair. Am J Physiol Heart Circ Physiol 2008, 295:H2113-H2127

22. Tabibiazar R, Cheung L, Han J, Swanson J, Beilhack A, An A, Dadras SS, Rockson N, Joshi S, Wagner R, Rockson SG: Inflammatory manifestations of experimental lymphatic insufficiency. PLoS Med 2006, 3:e254

23. Schirger A, Harrison EG Jr, Janes JM: Idiopathic lymphedema: review of 131 cases. JAMA 1962, 182:14-22

24. Mihara M, Hara H, Hayashi Y, Narushima M, Yamamoto T, Todokoro T, Iida T, Sawamoto N, Araki J, Kikuchi K, Murai N,
Okitsu T, Kisu I, Koshima I: Pathological steps of cancer-related lymphedema: histological changes in the collecting lymphatic vessels after lymphadenectomy. PLoS One 2012, 7:e41126

25. Unno N, Nishiyama M, Suzuki M, Tanaka H, Yamamoto N, Sagara D, Mano Y, Konno H: A novel method of measuring human lymphatic pumping using indocyanine green fluorescence lymphography. J Vasc Surg 2010, 52:946-952

26. Stanton AW, Modi S, Mellor RH, Levick JR, Mortimer PS: Recent advances in breast cancer-related lymphedema of the arm: lymphatic pump failure and predisposing factors. Lymphat Res Biol 2009, 7: 29-45

27. Liao S, von der Weid PY: Inflammation-induced lymphangiogenesis and lymphatic dysfunction. Angiogenesis 2014, 17:325-334

28. Swartz MA, Berk DA, Jain RK: Transport in lymphatic capillaries, I: macroscopic measurements using residence time distribution theory. Am J Physiol 1996, 270:H324-H329

29. Zaugg-Vesti B, Dorffler-Melly J, Spiegel M, Wen S, Franzeck UK, Bollinger A: Lymphatic capillary pressure in patients with primary lymphedema. Microvasc Res 1993, 46:128-134

30. Zhou Q, Wood R, Schwarz EM, Wang YJ, Xing L: Near-infrared lymphatic imaging demonstrates the dynamics of lymph flow and lymphangiogenesis during the acute versus chronic phases of arthritis in mice. Arthritis Rheum 2010, 62:1881-1889

31. Chakraborty S, Zawieja SD, Wang W, Lee Y, Wang YJ, von der Weid PY, Zawieja DC, Muthuchamy M: Lipopolysaccharide modulates neutrophil recruitment and macrophage polarization on lymphatic vessels and impairs lymphatic function in rat mesentery. Am J Physiol Heart Circ Physiol 2015, 309: H2042-H2057

32. Dieterich LC, Seidel CD, Detmar M: Lymphatic vessels: new targets for the treatment of inflammatory diseases. Angiogenesis 2014, 17 : $359-371$

33. Kim KE, Koh YJ, Jeon BH, Jang C, Han J, Kataru RP, Schwendener RA, Kim JM, Koh GY: Role of CD11b+ macrophages in intraperitoneal lipopolysaccharide-induced aberrant lymphangiogenesis and lymphatic function in the diaphragm. Am J Pathol 2009, $175: 1733-1745$

34. Huggenberger R, Siddiqui SS, Brander D, Ullmann S, Zimmermann K, Antsiferova M, Werner S, Alitalo K, Detmar M: An important role of lymphatic vessel activation in limiting acute inflammation. Blood 2011, 117:4667-4678

35. Liao S, Cheng G, Conner DA, Huang Y, Kucherlapati RS, Munn LL, Ruddle NH, Jain RK, Fukumura D, Padera TP: Impaired lymphatic contraction associated with immunosuppression. Proc Natl Acad Sci U S A 2011, 108:18784-18789

36. Zampell JC, Yan A, Elhadad S, Avraham T, Weitman E, Mehrara BJ: $\mathrm{CD} 4(+)$ cells regulate fibrosis and lymphangiogenesis in response to lymphatic fluid stasis. PLoS One 2012, 7:e49940

37. Ghanta S, Cuzzone DA, Torrisi JS, Albano NJ, Joseph WJ, Savetsky IL, Gardenier JC, Chang D, Zampell JC, Mehrara BJ: Regulation of inflammation and fibrosis by macrophages in lymphedema. Am J Physiol Heart Circ Physiol 2015, 308:H1065-H1077

38. Murray PJ, Wynn TA: Protective and pathogenic functions of macrophage subsets. Nat Rev Immunol 2011, 11:723-737

39. Zhang B, Wang J, Gao J, Guo Y, Chen X, Wang B, Gao J, Rao Z, Chen Z: Alternatively activated RAW264.7 macrophages enhance tumor lymphangiogenesis in mouse lung adenocarcinoma. J Cell Biochem 2009, 107:134-143 